Network Working Group

Request for Comments: 4994

Category: Standards Track
S. Zeng

B. Volz

K. Kinnear

Cisco systems, Inc. J. Brzozowski Comcast Cable September 2007

\title{
DHCPv6 Relay Agent Echo Request Option
}

Status of This Memo

This document specifies an Internet standards track protocol for the Internet community, and requests discussion and suggestions for improvements. Please refer to the current edition of the "Internet Official Protocol Standards" (STD 1) for the standardization state and status of this protocol. Distribution of this memo is unlimited.

\section{Abstract}

This memo defines a Relay Agent Echo Request option for the Dynamic Host Configuration Protocol for IPv6 (DHCPv6). The option allows a DHCPv6 relay agent to request a list of relay agent options that the server echoes back to the relay agent.

Table of Contents

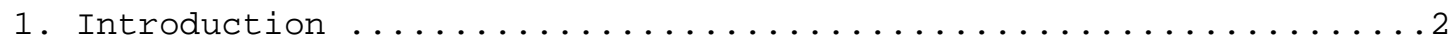

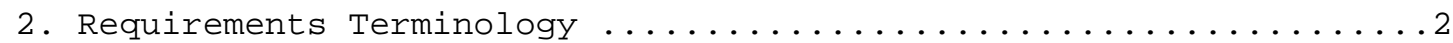

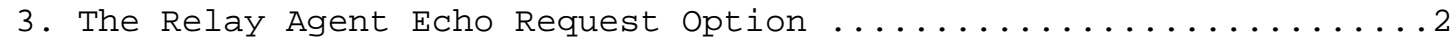

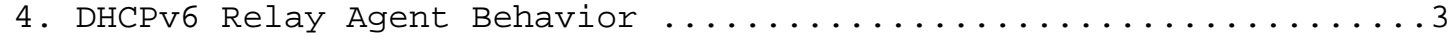

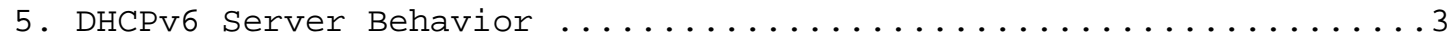

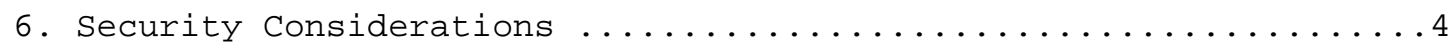

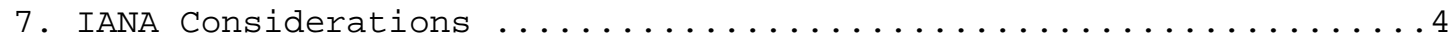

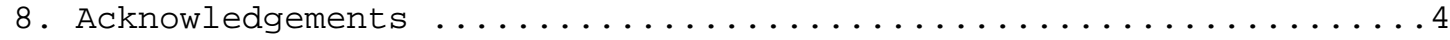

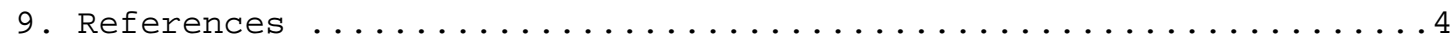

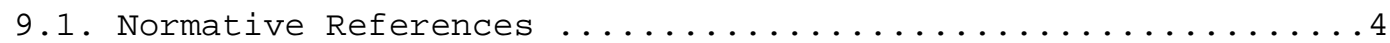

9.2. Informative References ...................... 


\section{Introduction}

DHCPv6 [2] provides a framework for configuring IPv6 clients with addresses and other network parameters. It includes a relay agent capability. A relay agent is an intermediary node that delivers DHCP messages between clients and servers. The relay agent and the server exchange information using options in relay agent messages. The relay agent may add relay agent options to the client DHCP message before forwarding it.

The information that relay agents supply can be used in the server's decision making about the addresses, delegated prefixes, and configuration parameters that the client is to receive. Likewise, the relay may need some of the information to efficiently return replies to clients.

In DHCPv4, the server generally echoes the relay agent option back verbatim to the relay agent in server-to-client replies [3]. However, DHCPv6 [2] does not require the server to do so. This could be problematic, as the relay agent may need to use some relay options even if the server does not recognize them.

This memo defines a relay agent echo request option that the relay agent uses to explicitly request a list of options that the server echoes back to the relay agent.

2. Requirements Terminology

The key words "MUST", "MUST NOT", "REQUIRED", "SHALL", "SHALL NOT", "SHOULD", "SHOULD NOT", "RECOMMENDED", "MAY", and "OPTIONAL" in this document are to be interpreted as described in [1].

3. The Relay Agent Echo Request Option

The relay agent adds options in the Relay Forward message that the server uses to guide its decision making with regard to address assignment, prefix delegation, and configuration parameters. The relay agent also knows which of these options that it will need to efficiently return replies to the client. It uses the relay agent Echo Request option to inform the server of the list of relay agent options that the server must echo back. 
The format of the DHCPv6 Relay Agent Echo Request option is shown below:

$\begin{array}{llllllllllllllllllllllllllllllllll}0 & 1 & 2 & 3 & 4 & 5 & 6 & 7 & 8 & 9 & 0 & 1 & 2 & 3 & 4 & 5 & 6 & 7 & 8 & 9 & 0 & 1 & 2 & 3 & 4 & 5 & 6 & 7 & 8 & 9 & 0 & 1\end{array}$

4. DHCPv6 Relay Agent Behavior

A relay agent MAY include an Echo Request option in a Relay Forward message to inform the server about options the relay agent wants the server to echo back to the relay agent. If the relay agent takes different actions based on whether an option is echoed back or not, then the relay agent SHOULD NOT include such an option in the Echo Request option. Note that the relay uses the OPTION_ORO [2] to request the server to return options (e.g., [4]) other than relay agent options in the Relay Forward message.

5. DHCPv6 Server Behavior

When a server creates a Relay-Reply, it SHOULD perform ERO processing after processing the ORO and other options processing. For each option in the ERO:

a. If the option is already in the Relay-Reply, the server MUST ignore that option and continue to process any remaining options in the ERO.

b. If the option was not in the received Relay-Forward, the server MUST ignore that option and continue to process any remaining options in the ERO.

c. Otherwise, the server MUST copy the option, verbatim, from the received Relay-Forward to the Relay-Reply, even if the server does not otherwise recognize that option. 
6. Security Considerations

As the Echo Request option is only exchanged between relay agents and DHCPv6 servers, section 21.1 of [2] provides details on securing DHCPv6 messages sent between servers and relay agents. And, section 23 of [2] provides general DHCPv6 security considerations.

7. IANA Considerations

IANA has assigned a DHCPV6 option code for the OPTION_ERO (Relay Agent Echo Request) Option (43).

8. Acknowledgements

Thanks to Ralph Droms, Josh Littlefield, Richard Johnson, and Hemant Singh for their consistent input, ideas, and review during the production of this document.

9. References

9.1. Normative References

[1] Bradner, S., "Key words for use in RFCs to Indicate Requirement Levels", BCP 14, RFC 2119, March 1997.

[2] Droms, R., Bound, J., Volz, B., Lemon, T., Perkins, C., and M. Carney, "Dynamic Host Configuration Protocol for IPv6 (DHCPv6)", RFC 3315, July 2003.

[3] Patrick, M., "DHCP Relay Agent Information Option", RFC 3046 , January 2001 .

\subsection{Informative References}

[4] Droms, R., Volz, B., and O. Troan, "DHCPv6 Relay Agent Assignment Notification (RAAN) Option", Work in Progress, November 2006 . 


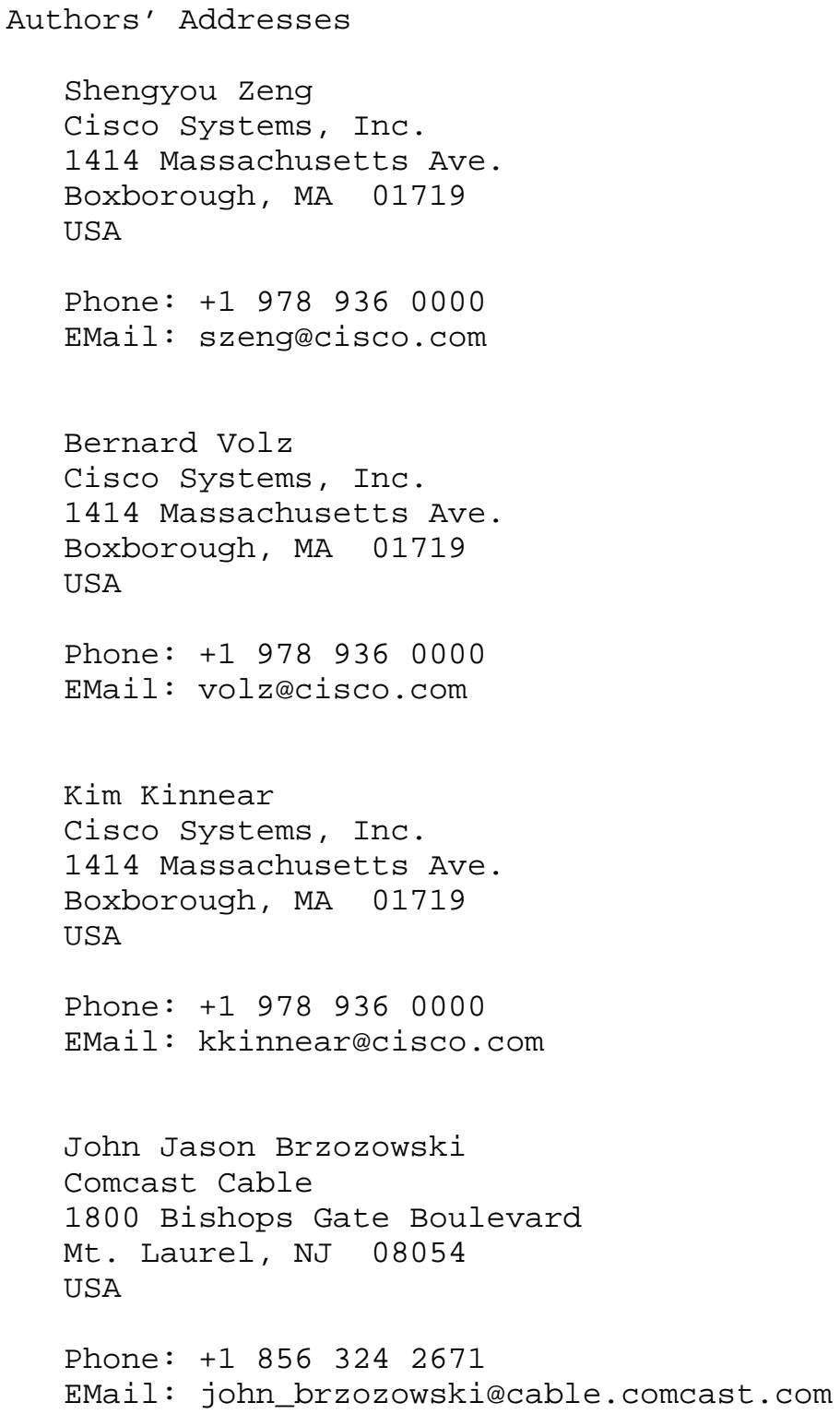


Full Copyright statement

Copyright (C) The IETF Trust (2007).

This document is subject to the rights, licenses and restrictions contained in BCP 78, and except as set forth therein, the authors retain all their rights.

This document and the information contained herein are provided on an "AS IS" basis and THE CONTRIBUTOR, THE ORGANIZATION HE/SHE REPRESENTS OR IS SPONSORED BY (IF ANY), THE INTERNET SOCIETY, THE IETF TRUST AND THE INTERNET ENGINEERING TASK FORCE DISCLAIM ALL WARRANTIES, EXPRESS OR IMPLIED, INCLUDING BUT NOT LIMITED TO ANY WARRANTY THAT THE USE OF THE INFORMATION HEREIN WILL NOT INFRINGE ANY RIGHTS OR ANY IMPLIED WARRANTIES OF MERCHANTABILITY OR FITNESS FOR A PARTICULAR PURPOSE.

Intellectual Property

The IETF takes no position regarding the validity or scope of any Intellectual Property Rights or other rights that might be claimed to pertain to the implementation or use of the technology described in this document or the extent to which any license under such rights might or might not be available; nor does it represent that it has made any independent effort to identify any such rights. Information on the procedures with respect to rights in RFC documents can be found in BCP 78 and BCP 79 .

Copies of IPR disclosures made to the IETF Secretariat and any assurances of licenses to be made available, or the result of an attempt made to obtain a general license or permission for the use of such proprietary rights by implementers or users of this specification can be obtained from the IETF on-line IPR repository at http://www.ietf.org/ipr.

The IETF invites any interested party to bring to its attention any copyrights, patents or patent applications, or other proprietary rights that may cover technology that may be required to implement this standard. Please address the information to the IETF at ietf-ipreietf.org. 